\title{
Observation of pentaquark resonances in $\Lambda_{b} \rightarrow J / \psi K^{-} p$ decays
}

\author{
B. Adeva ${ }^{1, a}$, on behalf of the LHCb Collaboration. \\ ${ }^{1}$ University of Santiago de Compostela, Spain.
}

\begin{abstract}
LHCb has recently reported the observation of exotic structures in the $J / \psi p$ channel, in $\Lambda_{b} \rightarrow J / \psi K^{-} p$ decay, which we discuss here and refer to as charmoniumpentaquark states. The data sample corresponds to an integrated luminosity of $3.0 \mathrm{fb}^{-1}$ acquired with the LHCb detector from 7 and $8 \mathrm{TeV} p p$ collisions. An amplitude analysis of the three-body final state reproduces the two-body mass and angular distributions. To obtain a satisfactory fit of the structures seen in the $J / \psi p$ mass spectrum, it is necessary to include two Breit-Wigner amplitudes that each describe a resonant state. Details are given on the significance, and prefered quantum numbers, of these two resonances.
\end{abstract}

\section{Selection of $\Lambda_{b} \rightarrow J / \psi K^{-} p$ at $\mathbf{L H C b}$}

The LHCb detector $[1,2]$ is one of the four major detectors at the Large Hadron Collider. It is instrumented in a cone arround the proton beam axis, covering the angles between 10 and $250 \mathrm{mrad}$ in the vertical plane, where most $b$ hadron decays produced in proton-proton collisions occur. The detector includes a high-precision tracking system with a dipole magnet, providing a measurement of momentum and impact parameter (IP), defined for charged particles as the minimum distance of a track to a primary $p p$ interaction vertex (PV). Different types of charged particles are distinguished using information from two ring-imaging Cherenkov detectors, a calorimeter and a muon system.

We discuss here data from $\Lambda_{b} \rightarrow J / \psi K^{-} p$ decay, corresponding to $1.0 \mathrm{fb}^{-1}$ of integrated luminosity acquired by LHCb at $\sqrt{s}=7 \mathrm{TeV}$, and $2.0 \mathrm{fb}^{-1}$ at $\sqrt{s}=8 \mathrm{TeV}$. Events are triggered by a $J / \psi \rightarrow \mu^{+} \mu^{-}$decay, with muon transverse momentum $p_{T}$ larger than $500 \mathrm{MeV}$. The dimuon system is required to form a vertex significantly displaced from the nearest $p p$ interaction vertex, and to have an invariant mass within $120 \mathrm{MeV}$ of the $J / \psi$ mass. After applying these requirements, there is a large $J / \psi$ signal over a small background. Only candidates with dimuon invariant mass between -48 and $+43 \mathrm{MeV}$ relative to the observed $J / \psi$ mass peak are selected, allowing for photon radiation.

Analysis preselection requirements are imposed prior to using a gradient boosted decision tree, BDTG [3], that separates the $\Lambda_{b}^{0}$ signal from backgrounds. The $p_{T}$ of individual particles must be larger than $550 \mathrm{MeV}$ for muons and $250 \mathrm{MeV}$ for hadrons. Each hadron must have a significantly non-zero impact parameter with respect to the $p p$ interaction vertex, and be positively identified in the particle ID system. The $K^{-} p$ system must form a vertex with $\chi^{2}<16$, as must the two muons from $J / \psi$ decay. In addition the $\Lambda_{b}^{0}$ candidate must form a good vertex, and the vector from the primary vertex to the $\Lambda_{b}^{0}$ vertex must align with the $\Lambda_{b}^{0}$ momentum so that the cosine of the angle between them is larger than 0.999 .

\footnotetext{
a e-mail: Bernardo.Adeva@usc.es
} 
The BDTG technique involves a "training" procedure using sideband data background and simulated signal samples of $2 \times 10^{6} \Lambda_{b} \rightarrow J / \psi K^{-} p$ events generated uniformly in phase space in the LHCb acceptance, using Pythia [4], with a special LHCb parameter tune [6], and the LHCb detector simulation based on Geant4 [7]. Specific backgrounds from $B_{s}^{0}$ and $B^{0}$ decays are vetoed, by removing combinations that when interpreted as $J / \psi K^{+} \pi^{-}$fall within $\pm 30 \mathrm{MeV}$ of the $B^{0}$ mass and when interpreted as $J / \psi K^{+} K^{-}$fall within $\pm 30 \mathrm{MeV}$ of the $B_{s}^{0}$ mass. After a relatively tight cut on the BDTG output variable, $26007 \pm 166$ signal candidates are selected, containing $5.4 \%$ background within $\pm 15 \mathrm{MeV}$ of the $J / \psi K^{-} p$ mas peak, as determined from a fit to the mass spectrum, shown in Fig. 1.

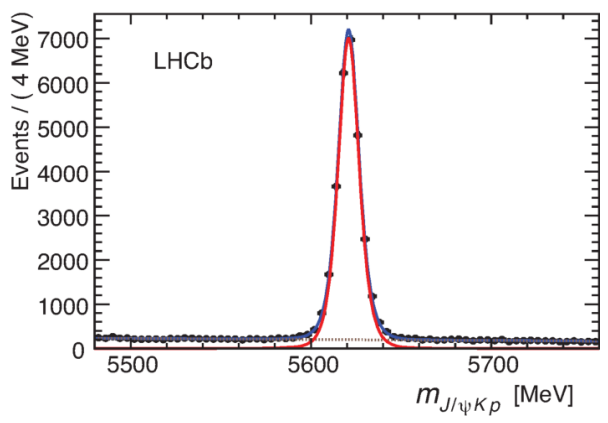

Figure 1. Invariant mass spectrum of $J / \psi K^{-} p$ combinations, with the total fit, signal, and background components shown as solid (blue), solid (red), and dashed lines, respectively.

\section{Summary of the $\Lambda_{b}^{0} \rightarrow J / \psi K^{-} p$ Dalitz plot analysis}

The large yields of $\Lambda_{b}^{0} \rightarrow J / \psi K^{-} p$ decays available at LHCb have been used earlier for a precise measurement of the $\Lambda_{b}^{0}$ lifetime [8]. Fig. 2 shows the Dalitz plot [9] using the $K^{-} p$ and $J / \psi p$ invariant masses-squared as independent variables. A distinct vertical band is observed in the $K^{-} p$ invariant mass distribution near $2.3 \mathrm{GeV}^{2}$ corresponding to the $\Lambda(1520)$ resonance. There is also a distinct horizontal band near $19.5 \mathrm{GeV}^{2}$. As structures are seen in both $K^{-} p$ and $J / \psi p$ mass distributions a full amplitude analysis is performed, using the available angular variables in addition to the mass distributions, in order to determine the resonances present. No structure is seen in the $J / \psi K^{-}$invariant mass.

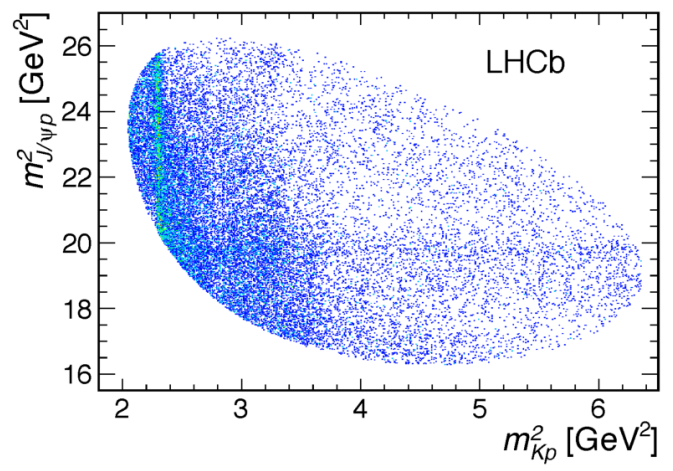

Figure 2. Invariant mass squared of $K^{-} p$ versus $J / \psi p$ for candidates within $\pm 15 \mathrm{MeV}$ of the $\Lambda_{b}^{0}$ mass.

The decay $\Lambda_{b}^{0} \rightarrow J / \psi K^{-} p$ can proceed by the diagram shown in Fig. 3 (a), and is expected to be dominated by $\Lambda^{*} \rightarrow K^{-} p$ resonances, as are evident in the data shown in Fig. 4 (a). It could also 
(a)

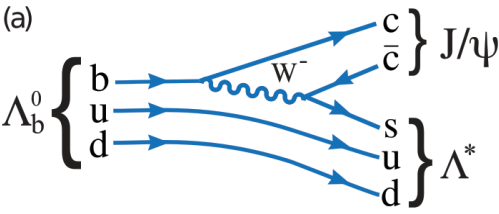

(b)

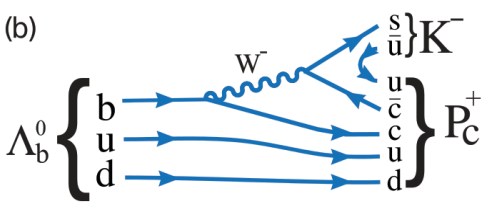

Figure 3. Feynman diagrams for the weak decay $\Lambda_{b}^{0} \rightarrow J / \psi K^{-} p$ in the Standard Model (a), and for the weak decay of $\Lambda_{b}^{0}$ allowing to form a pentaquark state (b).

have exotic contributions, as indicated by the diagram in Fig. 3 (b), which could result in resonant structures in the $J / \psi p$ mass spectrum shown in Fig. 3 (b).

In practice, resonances decaying strongly into $J / \psi p$ must have a minimal quark content of $c \bar{c} u u d$, and thus are charmonium pentaquarks. Such states have been labeled $P_{c}^{+}$, irrespective of the internal binding mechanism. In order to ascertain if the structures seen in Fig. 4 (b) are resonant in nature and not due to reflections generated by the $\Lambda^{*}$ states, it is necessary to perform a full amplitude analysis, allowing for interference effects between both decay sequences.

The analytical structure of the helicity amplitudes used is described in Section 3, and according to it a probability density is defined (PDF) that is a function of the six independent dimensions of the $J / \psi K^{-} p$ phase space, namely five decay angles (denoted collectively by $\Omega$ ) and the $K^{-} p$ invariant mass $m_{K p}$. The total PDF $\mathcal{P}\left(m_{K p}, \Omega \mid \vec{\omega}\right)$ also depends on a certain number of parameters $\vec{\omega}$ that include independent helicity amplitudes and resonance constants. The sum over all candidates $-2 \ln \mathcal{L}(\vec{\omega})=-2 \sum_{i} \ln \mathcal{P}$ is minimized to give the estimated values of the parameters $\vec{\omega}_{\text {min }}$, together with their covariance matrix. Such maximum likelihood fits are performed to the data, using different models of the amplitude analysis. The function $\mathcal{P}\left(m_{K p}, \Omega \mid \vec{\omega}\right)$ includes a background component with normalization fixed to be $5.4 \%$ of the total.
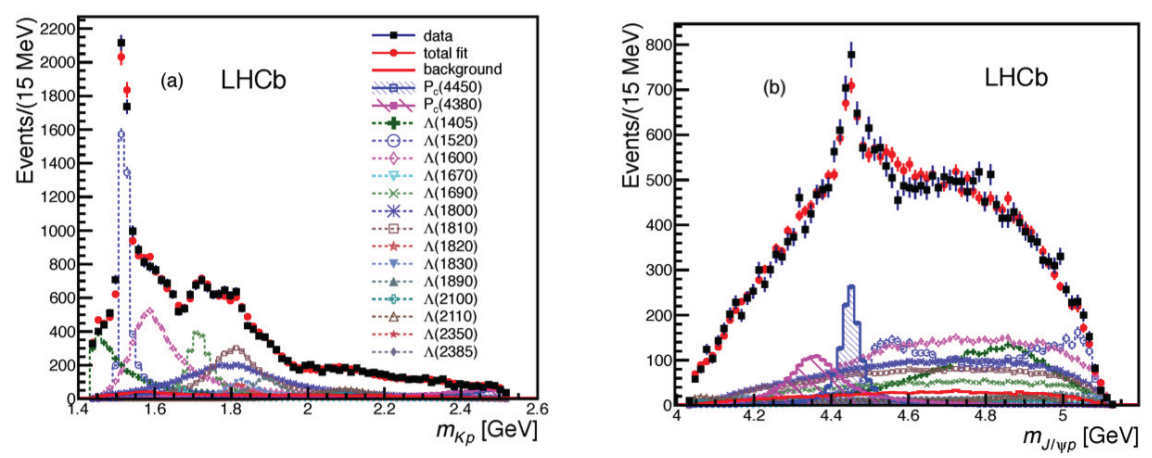

Figure 4. Fit projections for (a) $m_{K p}$ and (b) $m_{J / \psi p}$ for the reduced $\Lambda^{*}$ model with two $P_{c}^{+}$states. The data are shown as solid (black) squares, while the solid (red) points show the results of the fit. The solid (red) histogram shows the background distribution. The (blue) open squares with the shaded histogram represent the $P_{c}^{+}(4450)$ state, and the shaded histogram topped with (purple) filled squares represents the $P_{c}^{+}(4380)$ state. Each $\Lambda^{*}$ component is also shown. The error bars on the points showing the fit results are due to simulation statistics.

The strategy was to first try to fit the data with a model that can describe the mass and angular ditributions including only $\Lambda^{*}$ resonances, allowing all possible known states and decay amplitudes. We call this "extended" model. The masses and widths of the $14 \Lambda^{*}$ states are fixed to their PDG values, and the analysis is described in Ref. [13]. Variations of these parameters are considered in the systematic uncertainties. 


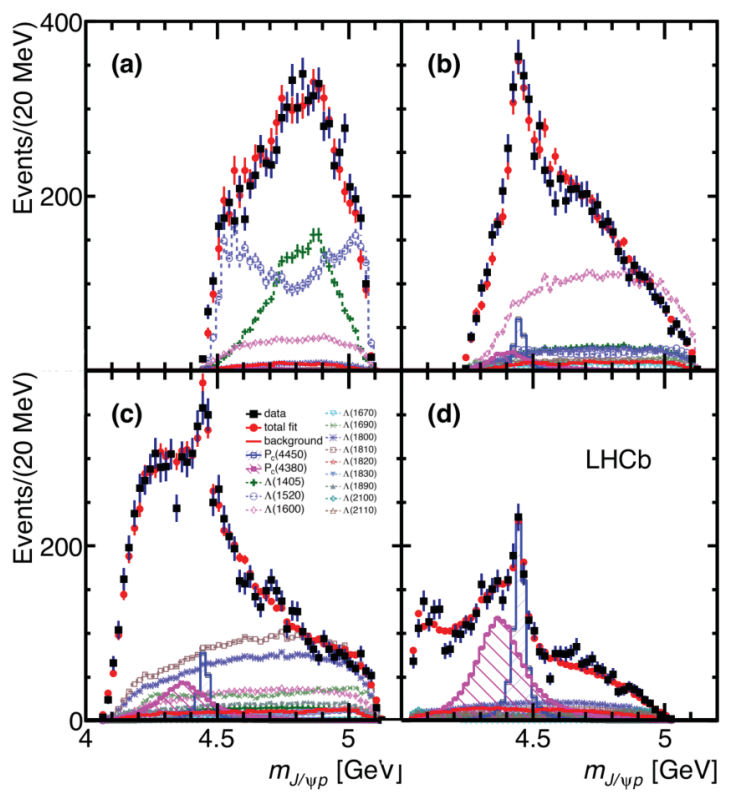

Figure 5. $m_{J / \psi p}$ in various intervals of $m_{K p}$ for the fit with the two $P_{c}^{+}$states:

(a) $m_{K p}<1.55 \mathrm{GeV}$,

(b) $1.55<m_{K p}<1.70 \mathrm{GeV}$,

(c) $1.70<m_{K p}<2.00 \mathrm{GeV}$, and

(d) $m_{K p}>2.00 \mathrm{GeV}$. The data are shown as

(black) squares with error bars, while the (red) circles show the results of the fit. The blue and purple histograms show the two $P_{c}^{+}$ states. See Fig.4 for the legend.

As this model did not give a satisfactory description of the data, one $P_{c}^{+}$state was added, and when that was not sufficient, a second state was included. When determining the parameters of the $P_{c}^{+}$states, a more restrictive model of the $K^{-} p$ states (referred to as "reduced" model) was used that includes only the $\Lambda^{*}$ resonances that are well motivated, and has fewer number of parameters. In both cases, the helicity couplings were expressed in terms of $L S$ couplings, where $L$ is the orbital angular momentum of the decay products and $S$ is their total spin. The number of $L S$ couplings used was also reduced when going from the extended model to the reduced model, which only kept the two lowest allowed values of $L$ for each resonance.

Using the extended model with one resonant $P_{c}^{+}$improves the fit quality, but it is still unacceptable (see Ref. [13] and supplemental material therein). Acceptable fits were only found with two $P_{c}^{+}$states. The central values of the fit results are given for the reduced $\Lambda^{*}$ model, and the differences in fitted quantities between both models are included in their systematic uncertainties.

The best fit combination finds two $P_{c}^{+}$states with $J^{P}$ values of $3 / 2^{-}$and $5 / 2^{+}$, for the lower and higher mass states, respectively. The fit projections are shown in Fig. 4, and both $m_{K p}$ and the peaking structure in $m_{J / \psi p}$ are reproduced by the fit. The two $P_{c}^{+}$states are found to have masses of $4380 \pm 8 \pm 29 \mathrm{MeV}$ and $4449.8 \pm 1.7 \pm 2.5 \mathrm{MeV}$, with corresponding widths of $205 \pm 18 \pm 86 \mathrm{MeV}$ and $39 \pm 5 \pm 19 \mathrm{MeV}$. The first uncertainty is statistical and the second is systematic. The mass resolution is approximately $2.5 \mathrm{MeV}$ and does not affect the width determinations. The angular distributions are reasonably well reproduced. A significant $\Lambda^{*}$ production recoiling against the $J / \psi$ is observed with the lowest mass contributions, the $\Lambda(1405)$ and $\Lambda(1520)$ states having fit fractions of $(15 \pm 1 \pm 6) \%$ and $(19 \pm 1 \pm 4) \%$, respectively.

A comparison of the fit results with the data in $m_{K p}$ intervals is particularly enlightening, and it is shown in Fig. 5. As $m_{K p}$ moves away from the $\Lambda^{*}$ resonances, the signal from both $P_{c}^{+}$states becomes clearer. In slice (d) of Fig. 5 both states form a large part of the mass spectrum. In fact, an interference effect is observed in the data as a consequence of the fact that the two $P_{c}^{+}$states have opposite parity, 
leading to an asymmetric distribution of the helicity angle in the $P_{c}^{+}$rest frame, i.e. the angle of the proton in $J / \psi p$ rest frame with respect to the $P_{c}^{+}$flight direction. This is illustrated in Fig. 6 for the entire $m_{K p}$ range, as described in Ref. [14]. The summed fit projections agree well with the angular distributions in the data showing that two interfering states are needed to reproduce the asymmetric distribution.

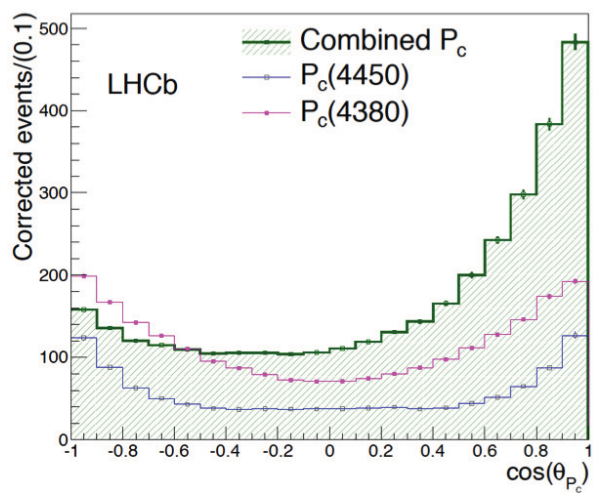

Figure 6. Distribution of the cosine of the $J / \psi$ angle in the $P_{c}^{+}$rest frame $\left(\theta_{P_{c}^{+}}\right)$. The interference between opposite parity $P_{c}^{+}$states is needed to explain the decay angular distribution. The fit projections are shown, indicating the combined signal (green), and the individually symmetric rates for each of the intefering amplitudes. Values of $\cos \theta_{P_{c}^{+}}$near -1 are correlated with values of $m_{K p}$ near threshold, while those near +1 are correlated with higher values.

The $-2 \ln \mathcal{L}$ values differ by only one unit between the best fit and the parity reversed combination $\left(3 / 2^{+}, 5 / 2^{-}\right)$. Other spin-parity combinations are less likely, although the $\left(5 / 2^{+}, 3 / 2^{-}\right)$pair changes $-2 \ln \mathcal{L}$ by only $2.3^{2}$ units and therefore cannot be ruled out. All combinations $1 / 2^{ \pm}$through $7 / 2^{ \pm}$were tested, and all others are disfavored by changes of more than $5^{2}$ in the $-2 \ln \mathcal{L}$ values.

Adding a single $5 / 2^{+} P_{c}^{+}$state to the fit with only $\Lambda^{*}$ states reduces $-2 \ln \mathcal{L}$ by $14.7^{2}$ using the extended model and adding a second lower mass $3 / 2^{-} P_{c}^{+}$state results in a further reduction of $11.6^{2}$. The combined reduction of $-2 \ln \mathcal{L}$ by the two states together is $18.7^{2}$. An independent calculation was performed by generating pseudoexperiments using the null hypothesis having amplitude parameters determined from the fits to the data with no or one $P_{c}^{+}$state. These studies show a reduction of the significances by about $20 \%$, giving overall significances of $9 \sigma$ and $12 \sigma$, for the lower and higher mass $P_{c}^{+}$states, respectively. The combined significance of two $P_{c}^{+}$states is $15 \sigma$.

\section{The multi-resonance model}

The two interfering processes shown in Fig. 1 produce distinct decay sequences: $\Lambda_{b}^{0} \rightarrow J / \psi \Lambda^{*}$, $\Lambda^{*} \rightarrow K^{-} p$ and $\Lambda_{b}^{0} \rightarrow P_{c}^{+} K^{-}, P_{c}^{+} \rightarrow J / \psi p$, with $J / \psi \rightarrow \mu^{+} \mu^{-}$in both cases. The helicity formalism is used [11], where each sequential decay $A \rightarrow B C$ contributes an amplitude term

$$
\mathcal{H}_{\lambda_{B}, \lambda_{C}}^{A \rightarrow B C} D_{\lambda_{A}, \lambda_{B}-\lambda_{C}}^{J_{A}}\left(\phi_{B}, \theta_{A}, 0\right)^{*} R_{A}\left(m_{B C}\right)=\mathcal{H}_{\lambda_{B}, \lambda_{C}}^{A \rightarrow B C} e^{i \lambda_{A} \phi_{B}} d_{\lambda_{A}, \lambda_{A}-\lambda_{B}}^{J_{A}}\left(\theta_{A}\right) R_{A}\left(m_{B C}\right),
$$

where $\lambda$ is the helicity (projection of the spin of the particle onto its momentum vector), and $\mathcal{H}_{\lambda_{B}, \lambda_{C}}^{A \rightarrow B C}$ are complex helicity-coupling amplitudes describing the decay dynamics. Here, $\theta_{A}$ and $\phi_{B}$ are the polar and azimuthal angles of $B$ in the rest frame of $A\left(\theta_{A}\right.$ is known as the helicity angle of $\left.A\right)$. The three angles of Wigner's $D$ matrix are Euler angles describing the rotation of the initial coordinate system with the $z$ axis along the helicity axis of $A$ to the coordinate system with the $z$ axis along the helicity axis of $B$. If $A$ has a non-negligible natural width, the invariant mass distribution of the $B$ and $C$ daughters is described by the complex function $R_{A}\left(m_{B C}\right)$ discussed below.

The helicity couplings can be further expressed in terms of $L S$ couplings $\left(B_{L S}\right)$, when the decay proceeds with orbital angular momentum $L$ and total spin $S$ of the $A$ and $B$ system. Using Clebsch- 
Gordan coefficients, the helicity couplings are expressed in terms of $L S$ couplings $\left(B_{L S}\right)$, where $L$ is the orbital angular momentum in the decay, and $S$ is the total spin of A plus B.

$$
\mathcal{H}_{\lambda_{B}, \lambda_{C}}^{A \rightarrow B C}=\sum_{L} \sum_{S} \sqrt{\frac{2 L+1}{2 J_{A}+1}} B_{L S}\left(\begin{array}{cc|c}
J_{B} & J_{C} & S \\
\lambda_{B} & -\lambda_{C} & \lambda_{B}-\lambda_{C}
\end{array}\right)\left(\begin{array}{cc|c}
L & S & J_{A} \\
0 & \lambda_{B}-\lambda_{C} & \lambda_{B}-\lambda_{C}
\end{array}\right) .
$$

Denoting $J / \psi$ as $\psi$, the matrix element for the $\Lambda_{b}^{0} \rightarrow J / \psi \Lambda^{*}$ decay sequence is

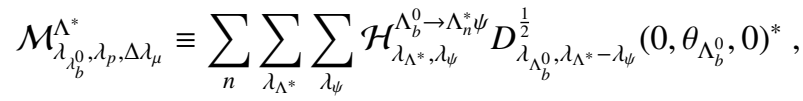

$$
\begin{aligned}
& \times \mathcal{H}_{\lambda_{p}, 0}^{\Lambda_{n}^{*} \rightarrow K p} D_{\lambda_{\Lambda^{*}, \lambda_{p}}}^{J_{\Lambda_{n}^{*}}}\left(\phi_{K}, \theta_{\Lambda^{*}}, 0\right)^{*} R_{\Lambda_{n}^{*}}\left(m_{K p}\right) D_{\lambda_{\psi}, \Delta \lambda_{\mu}}^{1}\left(\phi_{\mu}, \theta_{\psi}, 0\right)^{*},
\end{aligned}
$$

where the $x$ axis, in the coordinates describing the $\Lambda_{b}^{0}$ decay, is chosen to fit $\phi_{\Lambda^{*}}=0$. The sum over $n$ is due to many different $\Lambda_{n}^{*}$ resonances contributing to the amplitude. Since the $J / \psi$ decay is electromagnetic, the values of $\Delta \lambda_{\mu} \equiv \lambda_{\mu^{+}}-\lambda_{\mu^{-}}$are restricted to \pm 1 . There are four (six) independent complex $\mathcal{H}_{\lambda_{\Lambda^{*}}, \lambda_{n}^{0} \psi}^{\Lambda_{n}^{0} \Lambda_{*}^{*} \psi}$ couplings to fit for each $\Lambda_{n}^{*}$ resonance for $J_{\Lambda_{n}^{*}}=\frac{1}{2}\left(>\frac{1}{2}\right)$. They can be reduced to only one (three) free $B_{L S}$ coupling to fit if only the lowest (the lowest two) values of $L$ are considered. The mass $m_{K p}$, together with all decay angles entering Eq. 3, $\theta_{\Lambda_{b}^{0}}, \theta_{\Lambda_{n}^{*}}, \theta_{K}, \theta_{\psi}$, and $\theta_{\mu}$ (denoted collectively as $\Omega$ ), constitute the six independent dimensions of the $\Lambda_{b}^{0} \rightarrow J \psi p K^{-}$decay phase space.

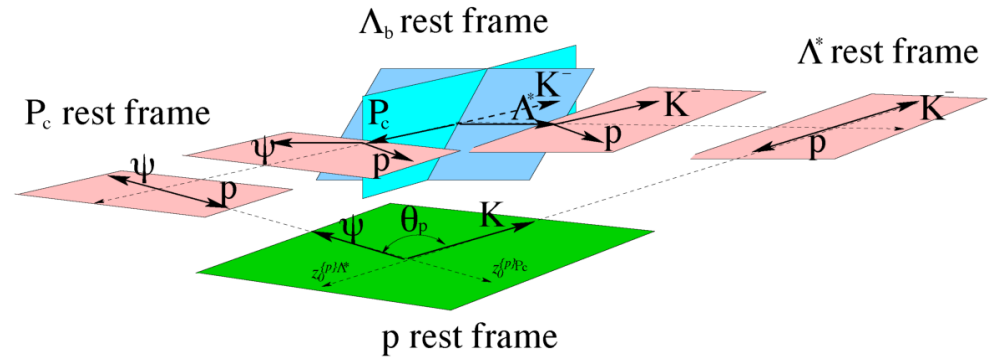

Figure 7. Definition of the decay angles in the $P_{c}^{+}$decay sequence.

Similarly, the matrix element for the $P_{c}^{+}$decay chain is given by

$$
\begin{array}{r}
\mathcal{M}_{\lambda_{\lambda_{b}^{0}}, \lambda_{p}^{P_{c}}, \Delta \lambda_{\mu}^{P_{c}}}^{P_{c}} \equiv \sum_{j} \sum_{\lambda_{P_{c}}} \sum_{\lambda_{\psi}^{P_{c}}} \mathcal{H}_{\lambda_{P_{c}}, 0}^{\lambda_{b}^{0} \rightarrow P_{c j} K} D_{\lambda_{b}^{0}, \lambda_{P_{c}}}^{\frac{1}{2}}\left(\phi_{P_{c}}, \theta_{\lambda_{b}^{0}}^{P_{c}}, 0\right)^{*}, \\
\times \mathcal{H}_{\lambda_{\psi}^{P_{c}}, \lambda_{p}^{P_{c}}}^{P_{c} \rightarrow{ }^{2}} D_{\lambda_{P_{c}}, \lambda_{\psi}^{P_{c}}-\lambda_{p}^{P_{c}}}^{J_{P_{c}}}\left(\phi_{\psi}, \theta_{P_{c}}, 0\right)^{*} R_{P_{c_{j}}}\left(m_{\psi p}\right) D_{\lambda_{\psi}^{P_{c}}, \Delta \lambda_{\mu}^{P_{c}}}^{1}\left(\phi_{\mu}^{P_{c}}, \theta_{\psi}^{P_{c}}, 0\right)^{*},
\end{array}
$$

where the angles and helicity states carry the superscript or subscript $P_{c}$ to distinguish them from those defined for the $\Lambda^{*}$ decay chain. The sum over $j$ allows for the possibility of contributions from more than one $P_{c}$ resonances. There are two (three) independent helicity couplings $\mathcal{H}_{\lambda_{\psi}^{P_{c}}, \lambda_{p}^{P_{c}}}^{P_{c j} \rightarrow \psi p}$ for $J_{P_{c j}}=\frac{1}{2}\left(>\frac{1}{2}\right)$, and a ratio of the two $\mathcal{H}_{\lambda_{P_{c}}, 0}^{\Lambda_{b}^{0} \rightarrow P_{c j} K}$ couplings to determine from the data.

The mass-dependent $R_{\Lambda_{n}^{*}}\left(m_{K p}\right)$ and $R_{P_{c j}}\left(m_{J \psi p}\right)$ terms are given by

$$
R_{X}(m)=B_{L_{\Lambda_{b}^{0}}^{\prime}}^{\prime}\left(p, p_{0}, d\right)\left(\frac{p}{M_{\Lambda_{b}^{0}}}\right)^{L_{\Lambda_{b}^{0}}^{X}} \times B W\left(m \mid M_{0 X}, \Gamma_{0 X}\right) B_{L_{X}^{\prime}}^{\prime}\left(q, q_{0}, d\right)\left(\frac{q}{M_{0 X}}\right)^{L_{X}} .
$$


Here $p$ is the $X=\Lambda^{*}$ or $P_{c}^{+}$momentum in the $\Lambda_{b}^{0}$ rest frame, and $q$ is the momentum of either decay product of $X$ in the $X$ rest frame. The symbols $p_{0}$ and $q_{0}$ denote values of these quantities at the resonance peak $\left(m=M_{0 X}\right)$. The orbital angular momentum between the decay products of $\Lambda_{b}^{0}$ is denoted as $L_{\Lambda_{b}^{0}}^{X}$. Similarly $L_{X}$ is the orbital angular momentum between the decay products of $X$. The orbital angular momentum barrier factors $p^{L} B_{L}^{\prime}\left(p, p_{0}, d\right)$ involve the Blatt-Weisskopf functions, and account for the difficulty in creating larger orbital angular momentum $L$, which depends on the momentum of the decay products $p$ and on the size of the decaying particle, given by the $d$ constant. The value $d=3.0 \mathrm{GeV}^{-1} \sim 0.6 \mathrm{fm}$ was set. The relativistic Breit-Wigner amplitude is given by

$$
B W\left(m \mid M_{0 X}, \Gamma_{0 X}\right)=\frac{1}{M_{0 X}^{2}-m^{2}-i M_{0 X} \Gamma(m)},
$$

where

$$
\Gamma(m)=\Gamma_{0 X}\left(\frac{q}{q_{0}}\right)^{2 L_{X}+1} \frac{M_{0 X}}{m} B_{L_{X}}^{\prime}\left(q, q_{0}, d\right)^{2}
$$

is the mass-dependent width of the resonance. For the $\Lambda(1405)$ resonance, which peaks below the $K^{-} p$ threshold, a Flatté-like parametrization [12] is used, with appropriate values of the couplings for the allowed channels $\Sigma \pi$ and $K p$.

To properly describe the interference between the $\Lambda^{*}$ and $P_{c}^{+}$amplitudes, the proton and muon helicity states must be rotated from the $\Lambda^{*}$ to the $P_{c}^{+}$rest frames. In the proton case, the rotation angle is $\theta_{p}$ (see Fig. 7), and no azimuth is involved since the $\Lambda^{*}$ and the $P_{c}^{+}$decay in the same plane. In the muon case, an azimuthal rotation about the $J / \psi$ flight direction, by an angle $\alpha_{\mu}$, is implied in the transformation between the above reference frames, the polar angle being absent just because the $\mu^{+}$ comes from $J / \psi$ decay in both reference frames. The resulting expression is

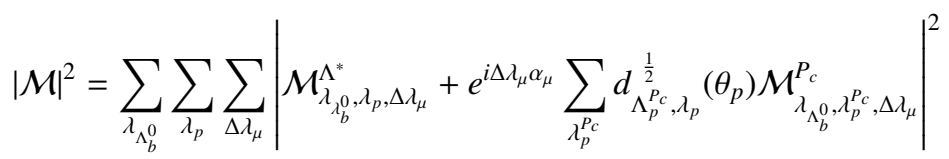

where $\Delta \lambda_{\mu}= \pm 1$ accounts for both muon spin-aligned orientations. Parity conservation in $\Lambda_{b}^{0}$ production at the LHC (in absence of polarized beams) prevents $\Lambda_{b}^{0}$ longitudinal polarization, so $\lambda_{\Lambda_{b}^{0}}=+1$ and $\lambda_{\Lambda_{b}^{0}}=-1$ are equally likely, which is reflected in Eq. 2.



Figure 8. Fitted values of the real and imaginary parts of the amplitudes for the baseline $\left(3 / 2^{-}, 5 / 2^{+}\right)$fit for (a) the $P_{c}(4450)^{+}$state and (b) the $P_{c}(4380)^{+}$state, each divided into six $m_{J / \psi p}$ bins of equal width between $-\Gamma_{0}$ and $+\Gamma_{0}$ shown in the Argand diagrams as connected points with error bars $\left(m_{J / \psi p}\right.$ increases counterclockwise). The solid (red) curves are the predictions from the Briet-Wigner formula in Eq. 1 evaluated for the fitted values of the mass and width in each case.

The matrix element given by Eq. 2 is a six dimensional function of $m_{K p}$ and $\Omega$ and depends on the fit parameters $\vec{\omega}$, which represent independent helicity or $L S$ couplings, and masses and widths of resonances $\mathcal{M}=\mathcal{M}\left(m_{K p}, \Omega \mid \vec{\omega}\right)$. After accounting for the selection efficiency, the probability density function (PDF) described in Section 2 is obtained. 


\section{Further evidence of resonant character}

The resonant character of the higher mass, narrower $P_{c}^{+}$state is further explored by viewing the evolution of the $m_{J / \psi p}$ dependent part of the Breit-Wigner amplitude discussed above for the $P_{c}(4450)^{+}$, in formula 1. Its magnitude and phase vary with $m_{J / \psi p}$ according to an approximately circular trajectory, as it is shown in Fig. 8 (a). The resulting Argand diagram is consistent with a rapid counterclockwise change of the $P_{c}(4450)^{+}$phase when its magnitude reaches the maximum, a behavior characteristic of a resonance. A similar study for the wider state is also shown in Fig. 8 (b), where the fit also shows a large phase change, the amplitude values being sensitive to the details of the $\Lambda^{*}$ model, and so this latter study is not conclusive.

\section{Summary}

In conclusion, a full amplitude fit has been presented to the $\Lambda_{b}^{0} \rightarrow J / \psi K^{-} p$ decay. A significant $\Lambda^{*}$ production recoiling against the $J / \psi$ is observed, and the data cannot be satisfactorily described without including two Breit-Wigner shaped resonances in the $J / \psi p$ invariant mass distribution. The significances of the lower mass and higher mass states are 9 and 12 standard deviations, respectively. These structures cannot be accounted for by reflections from $J / \psi \Lambda^{*}$ resonances or other known sources. Interpreted as resonant states they must have minimal quark content $c \bar{c} u u d$, and would therefore be called charmonium pentaquark states. The lighter state $P_{c}(4380)^{+}$has a mass of $4380 \pm 8 \pm 29 \mathrm{MeV}$ and a width of $205 \pm 18 \pm 86 \mathrm{MeV}$, while the heavier state $P_{c}(4450)^{+}$has a mass of $4449.8 \pm 1.7 \pm 2.5 \mathrm{MeV}$ and a width of $39 \pm 5 \pm 19 \mathrm{MeV}$. The parities of the two states are opposite with the preferred spins being $3 / 2$ for one state and $5 / 2$ for the other.

\section{References}

[1] LHCb collaboration, Alves, A.A. Jr et al., JINST 3, S088005 (2008)

[2] LHCb collaboration, Aaij et al. Int. J. Mod. Phys. A30, 1530022 (2015).

[3] A. Hoecker et al., Proc. Sci. ACAT (2007) 040.

[4] T. Sjöstrand, S. Mrenna and P. Skands, Comput. Phys. Commun. 178 (2008) 852.

[5] D. J. Lange, Nucl. Inst. Meth. A462 (2001) 152.

[6] I. Belyaev et al., J. Phys. Conf. Ser. 331 (2011) 032047.

[7] S. Agostinelli et al., Geant4 Collaboration. Nucl. Inst. Meth. Phys. Res. Sect. A 506, 250 (2003).

[8] R. Aaij et al., LHCb Collaboration, Phys. Rev. Lett. 111, 102003 (2013).

[9] R. H. Dalitz, Philos. Mag. Ser. 5 44, 1068 (1953).

[10] K. A. Olive et al. (Particle Data Group) Review of Particle Physics, Chin. Phys. C 38, 090001 (2014).

[11] S. U. Chung, CERN Report, CERN-71-08; J. D. Richman, CALT-68-1148; M. Jacob and G. C. Wick, Ann. Phys. (N.Y.) 7, 404 (1959).

[12] S. M. Flatté, Phys. Lett., 63B, 224 (1976).

[13] R. Aaij et al. (LHCb Collaboration), "Observation of $J / \psi p$ resonances consistent with pentaquark states in $\Lambda_{b}^{0} \rightarrow J / \psi K^{-} p$ decays ”,Phys. Rev. Lett. 115, 072001 (2015), 12 Aug. 2015.

[14] S. Stone, Meeting of the APS Division of Particles and Fields, DPF Ann Arbor, DPF2015-457, August 2015, arXiv:1509.04051 